\title{
Combined channel gain and QoS-based access-aware cell selection in LTE-advanced HetNets
}

\begin{abstract}
LTE-Advanced HetNets deployment addresses issues of growing quality of service (QoS) demand, high data rates, and expanding coverage. Among the core challenges is load balancing due to user equipments (UEs) offloading for association using received signal received power (RSRP). The low-power tier is under-utilized whereas, UEs having poor channel quality consume significant amount of available resources in the network. The proposed combined channel gain and quality of service (QoS)-based access-aware (CCGQA) cell selection scheme emphasizes on the importance of combined metrics to achieve improved performance through load balancing in HetNets. The QoS requirements of the high priority UEs (HPUs) can be satisfied through association with the tier giving maximum channel gain. The association for non-QoS traffic, low priority UEs (LPUs) is by the joint metrics (channel gain, channel access probabilities of LPUs and HPUs), while the remaining resources allocated to the LPUs. The CCGQA scheme has 1.72 times UEs connected to PeNBs, $8 \%$ enhanced loadbalancing fairness compared with the RSRP and RSRP+6dB bias cell selections. The average UE throughput of LPUs has improved by $73.7 \%$ using the proposed CCGQA after satisfying the QoS requirements of HPUs.
\end{abstract}

Keyword: LTE advanced; Cell range expansion; Picocell; Hybrid cell association; Load balancing; Heterogeneous networks 